\title{
Assessment of the behavior of children in painful situations: literature review
}

\author{
Luciana Leonetti Correia, ${ }^{1}$ Maria Beatriz Martins Linhares ${ }^{2}$
}

\begin{abstract}
Objective: To analyze the scientific production published between 2001 and 2006 describing studies assessing children in painful situations, with a focus on preschool children.

Sources: Searches were run for empirical articles indexed in electronic databases using the keywords pain assessment and behavior and their equivalents in Portuguese.

Summary of the findings: A total of 33 articles were identified, 18 of which were studies of the psychometric properties of instruments for the measurement of pain, while 11 dealt with the application of such instruments and four were studies of intervention procedures for the relief of pain.

Conclusions: The results indicate that more recent literature on the assessment of preschool children's behavior in painful situations is focused on testing the psychometric properties of measurement instruments. Acute pain, especially during surgical procedures, was most often assessed using one-dimensional instruments. Pain in children was most often assessed using facial activity as indicator and intensity as dimension. With relation to assessment of pain in special populations, advances were observed related to assessment of pain in preverbal children or preschool children with special needs.
\end{abstract}

J Pediatr (Rio J). 2008;84(6):477-486: Pain measurement, behavior, preschool.

\section{Introduction}

According to the International Association for the Study of Pain (IASP), pain is an "unpleasant sensory and emotional experience associated with actual or potential tissue damage or described in terms of such damage. ${ }^{1}$ However, this definition is incomplete to the extent that it does consider that babies and people with cognitive deficits feel pain without being capable of describing the pain that they feel.

Pain can be classified as acute, when it is the result of symptoms, and as such its biological function is as a warning to the organism. This type of pain is related to neurovegetative changes and is delineated spatiotemporally to the condition that causes it, such as, for example, inflammations, traumas, infections or postoperative recovery. Chronic pain, in turn, is the result of chronic pathological processes affecting somatic structures or of prolonged central nervous system dysfunction. When the symptom pain is chronic, it becomes a disease, and treatment is, generally, complex and tailored to the individual, as is the case with oncological and neuropathic pain and also with arthritis and fibromyalgia. ${ }^{2}$

The dimensions in which pain is evaluated may be of the following types: intensity, location, duration and affective quality. ${ }^{3}$ The measures used to evaluate pain, in turn, correspond to self-reported measures and behavioral observations and to physiological measures, such as heart rate and blood pressure; the second group being considered supplementary measures to the first. There are other variables which are of significance to the interaction between pain indicators and which can modulate the perception, intensity, sensation

1. Doutoranda, Programa de Pós-Graduação em Saúde Mental, Faculdade de Medicina de Ribeirão Preto, Universidade de São Paulo (USP), Ribeirão Preto, SP, Brazil.

2. Doutora. Professora, Departamento de Neurociências e Ciências do Comportamento, Faculdade de Medicina de Ribeirão Preto, USP, Ribeirão Preto, SP, Brazil.

No conflicts of interest declared concerning the publication of this article.

Suggested citation: Correia LL, Linhares MB. Assessment of the behavior of children in painful situations: literature review. J Pediatr (Rio J). 2008;84(6): 477-486.

Manuscript received Apr 14 2008, accepted for publication Jul 232008.

doi:10.2223/JPED.1830 
and quality of pain experienced, such as, for example, cognition, emotion and motivation, in addition to variables relating to the environmental context. ${ }^{4}$

Accurate evaluation of pain experienced requires behavioral and physiological measures that evaluate different aspects of the experience of pain, such as intensity, location, pattern, context and significance. ${ }^{4}$ The choice of an appropriate method for clinical assessment of pain should be based on the type of pain and the child's medical condition, and it is necessary to verify child's age and level of cognitive and emotional development. ${ }^{5}$ Self-reporting is considered a reliable measure for the evaluation of both the presence and intensity of pain. ${ }^{3}$

Self-reporting is based on the patient's ability to communicate their symptoms. From 2 years of age onwards children are able to provide information about their pain, such as its location, for example; however, at 2 years of age they rarely understand the concepts related to the intensity and quality of their pain. ${ }^{2}$ Preverbal children, or those incapable of communicating verbally, are limited in terms of self-reporting and so pain assessment by parents and/or caregivers is an important source of information for the evaluation of pain in this population.

When choosing pain measurement instruments, one should be alert to their psychometric qualities, so that the following parameters are met: validity (whether the instrument evaluates the construct/concept it is meant to evaluate), reliability (the agreement or consistency of results when the instrument is used repeatedly under similar conditions or when evaluations are carried out by independent examiners), sensitivity (how good a test is at identifying individuals who do exhibit that which is being tested for), specificity (how good a test is at identifying individuals who do not exhibit that which is being tested for) and clinical applicability (the usefulness of the measurement in the clinical environment in which it will be used). ${ }^{6}$

Pain measurement instruments can express their results in four types of scale: nominal scales, which merely serve to name, identify and/or categorize (pain vs. no pain); ordinal scales, which order the object of study according to certain characteristics of comparison (no pain, mild pain, moderate pain and strong pain); interval scales, with fixed intervals between numbers making it possible to compare differences between measurements, which are relative (pain thermometer in centimeters); and ratios, which offer an absolute zero and therefore make it possible to determine the absolute magnitude of the measurement in question. The last of these types is the most often used in experimental situations when assessing pain in adults. ${ }^{6}$

Since the need to measure pain in children and the difficulties involved with obtaining precise indicators of pain in small preschool children have been established, this literature review was proposed. The objective is to carry out a critical analysis of the designs and methodology of empirical studies published in indexed periodicals between 2001 and 2006 on the subject of the behavior of preschool children associated with pain.

\section{Sources}

Searches were run for empirical articles indexed in electronic databases (PubMed, PsycINFO and LILACS), using the keywords pain assessment and behavior and their equivalents in Portuguese, avaliação de dor and comportamento.

The initial inclusion criteria were defined by setting the following limits to the searches run on PubMed, PsycINFO and LILACS: articles published from 2001 to 2006, in the indexed literature on empirical studies, in the languages English, Spanish or Portuguese, carried out exclusively with humans aged 2 to 5 years.

This first procedure identified 78 articles. After reading the abstracts, studies were excluded if their objective was not specifically related to the subject of pain and behavior (31 articles), if they were review papers ( 8 articles) or if they were case studies ( 1 article), resulting in a total of 40 articles removed from the list. Thirty-three of the remaining 38 articles were located and analyzed.

\section{Synthesis of data}

The 33 articles that were located were classified into three groups, specifically: studies into the psychometric properties of pain measurement instruments $(n=18)$, studies of the application of pain measurement instruments $(n=11)$ and studies evaluating intervention procedures for pain relief $(n=4)$.

\section{Studies of the psychometric properties of pain measurement instruments}

Of the 18 studies about evaluation of the psychometric properties of pain measurement instruments, 15 investigated acute pain, while just three studies assessed both acute pain and chronic pain. This demonstrates that the great majority of studies focused on evaluating instruments that were aimed more at acute pain than chronic pain. Chronic pain is difficult to identify due to the more prolonged duration, in contrast with acute pain, which is characterized by being focused and fixed in time.

\section{Measurement scales of the instruments analyzed}

It was notable that just two of the instruments studied the Cardiac Analgesic Assessment Scale (CAAS) ${ }^{7}$ and the Toddler-Preschooler Postoperative Pain Scale (TPPPS) ${ }^{8,9}$ - use interval scales, i.e. scales that offer a more precise measurement, since there is a unit of measurement which has a constant interval all along the scale. ${ }^{6}$ The CAAS is a scale 
specifically for the context of postoperative care of child cardiac patients, which assesses pain based on physiological indicators (size of pupil, heart rate and blood pressure) and on behavior (body activity). ${ }^{7}$ The TPPPS, in turn, is a scale that can be used to assess pain during and after medical or surgical procedures, on the basis of behavioral indicators. ${ }^{8,9}$

In the remainder of the studies, the majority of the instruments whose psychometric qualities were being assessed used ordinal scales, i.e., they categorize attributes and establish a sequential order based on the characteristics of the attribute measured. ${ }^{6}$ It should be pointed out that, although ordinal scales are less precise than interval scales, they are generally used with children to obtain self-reported pain assessments since they are easier for children to understand, as is the case of the Oucher Faces Scale, ${ }^{10}$ the Faces Pain Scale $(\text { FPS })^{11,12}$ and the Facial Affective Scale (FAS). ${ }^{11}$

Nevertheless, in the course of this literature review it was found that ordinal scales are also used by trained observers or by the medical and nursing teams to assess child pain. In these cases the following instruments were used: the Objective Pain Scale (OPS), ${ }^{8}$ the Faces, Leg, Activity, Cry and Consolability scale (FLACC), ${ }^{8,13-15}$ the Children's Hospital of Eastern Ontario Pain Scale (CHEOPS), ${ }^{8}$ the Pediatric Pain Profile (PPP), ${ }^{16}$ the Alder Hey Triage Pain Score (AHTPS), ${ }^{17}$ the Child Facial Coding System (CFCS) ${ }^{18}$ and the Non-Communicating Children's Pain Checklist-Revised (NCCPC-R). ${ }^{19}$

\section{Contexts of pain assessment}

With relation to the use of instruments validated for specific pain contexts, of note are the CAAS for pain assessment in cardiac postoperative care ${ }^{7}$ and the AHTPS for triage at emergency services. ${ }^{17}$ Both of these scales, to the extent that they were validated in these specific contexts, are more appropriate when used exclusively in those situations.

Nevertheless, in the majority of the studies, the psychometric properties of different instruments were evaluated in a diverse range of contexts and involved different samples. Of all of the scales, the FLACC is of interest since it has been widely used in clinical-hospital contexts and has been used both with preverbal children, ${ }^{15}$ and with children at speaking age, with typical ${ }^{8,9}$ and atypical development. ${ }^{13,14}$

In addition to the FLACC, a further 12 instruments for pain assessment of children in a clinical-hospital context were identified. Three are of note for the specific contributions of three instruments. The TPPPS was the scale that best discriminated between the states of pain and no pain, when compared with the FLACC and the COMFORT scales, specifically with respect to postoperative pain assessment. ${ }^{9}$ The Parent's Postoperative Pain Measure (PPPM), validated for the postoperative phase, can also be administered by parents to assess the pain of their children after hospital discharge,${ }^{20}$ which has meant that multiple informants (caregivers) can take part in assessing the perceived pain of children. Finally, the Brief Behavioral Distress Scale (BBDS), in contrast with the other scales, allows for child patients' symptoms of pain and stress to be assessed in combination. ${ }^{21}$

Another feature that was observed is that a single instrument can be employed to assess pain in different contexts. The FPS, for example, has been validated to be administered in the clinical hospital context of surgical procedures ${ }^{11}$ and invasive blood collection procedure, ${ }^{21}$ during immunization, ${ }^{18}$ ear piercing ${ }^{22}$ and for the assessment of remembered painful situations. ${ }^{19}$

\section{Phases of pain assessment}

The majority of studies related to psychometric properties used follow-up with repeated measures. However, six studies performed baseline assessments before a painful procedure, ${ }^{8,10,16,18,21,23}$ which indicates a more robust methodology than the others, particularly when using an ordinal measure. Knowledge of baseline pain allows for better pain assessment based on comparison with points in time after the painful procedure. Three of these six studies assessed pain in the context of a surgical procedure, ${ }^{8,10,16}$ one in the context of an invasive blood collection procedure, ${ }^{21}$ another during immunization ${ }^{18}$ and one in the situation of remembered painful episodes. ${ }^{19}$

Specifically with relation to the studies related to surgical procedures, eight defined their objectives as assessing pain during the postoperative period; not including a baseline pain assessment. ${ }^{7,9,11,13-15,20,21}$ These studies performed either two or three postoperative pain assessments, and there was a great deal of variation in terms of the time when assessments were carried out. The multiple-assessment approach is highly to be recommended in postoperative pain assessment, since it offers better monitoring of the clinical progress of the patient's pain during this period, in contrast with acute pain, which is focused.

Two studies investigated postoperative administration of analgesia, ${ }^{7,21}$ using samples with randomized groups, in order to verify the sensitivity of the CAAS and PPPM instruments to the effects of the analgesia. In both studies the instruments proved sensitive enough to detect changes in the children's pain levels, as a result of the response to the analgesia used over time.

\section{Assessment of pain indicators and dimensions}

In the studies reviewed here, the pain dimensions assessed by the instruments were intensity and affective quality of pain. The intensity of pain was assessed using indicators of behavioral or physiological changes, or a combination of these. The CAAS, ${ }^{7}$ the OPS $^{8}$ and the NCCPC- ${ }^{19}$ are instruments that combine indicators- behavioral and physiological - and focus on the intensity of pain. 
In the case of instruments that assessed pain exclusively based on indicators of behavioral change (TPPPS, AHTPS and CHEOPS), these focus on a combination of three aspects of behavior, namely: the child's corporal, verbal and facial expressions. The PPPM, the FLACC and the PPP only assess facial expressions combined with body movements. Yet other instruments only take account of changes in facial expression, such as the Oucher Faces Scale, the Wong-Baker Faces Scale, the CFCS and the FPS.

Just one study combined assessment of the pain dimension intensity with the dimension affective quality, by using two different instruments, respectively the FPS and the FAS. ${ }^{11}$ The FAS only assessed the affective magnitude of pain using nine faces that range in expression from happiness to sadness. The authors state that the reason for using the FAS is to differentiate the affective dimension of pain from its sensory dimension. However, using that scale could confound the specific assessment of pain, since a happy face may not exactly correspond to an absence of pain. The child may not choose the initial face despite not feeling pain if they are also not happy. This scale inappropriately superimposes two different constructs, i.e., pain and happiness/sadness.

\section{The psychometric properties assessed}

Firstly, it should be emphasized that this is a review with limits of time from 2001 to 2006 and therefore only includes the psychometric properties of those scales which have been described in studies published within this period.

As the studies were analyzed it became clear that, validity was the psychometric parameter most often evaluated. Validity is of extreme importance, since instruments that have not undergone validity studies do not provide precise and reliable data and, therefore, should not be used to measure pain.

With relation to the psychometric properties investigated in the studies on pain scales, five scales are of note, namely: the FLACC, the TPPPS, the CHEOPS, the OPS and the PPP. These scales met all of the psychometric parameters of validity, reliability, sensitivity, specificity and clinical applicability. Due to these characteristics, these scales offer great advantages in terms of measurement power in relation to the other scales for pain assessment of preschool children with typical development.

Furthermore, the FLACC is the only scale that, in addition to being widely used in studies with samples of typical populations, also had its psychometric properties investigated with samples of populations with atypical development ${ }^{13,14}$ and with preverbal children. ${ }^{15}$

The psychometric properties of four of the scales (FLACC, CHEOPS, OPS and TPPPS) were investigated when administrated by trained examiners who assessed the pain of children aged 1 to 5 years at four distinct pain assessment times (before and after surgery, before analgesia and during admission to the ward). ${ }^{8}$ In that study, the CHEOPS had better psychometric results when compared to the other scales for the pain assessment times described above. It should be pointed out that the CHEOPS is a postoperative pain assessment scale and that it can also be used to monitor the efficacy of interventions to relieve pain and discomfort.

\section{The relevance of cultural factors}

Studies that adapt scales for different cultures are important from a methodological point of view, since the response to pain may be affected by cultural differences. In this review, it was found that the Oucher Faces Scale was the only instrument which had three specific versions aimed at different populations, namely: Caucasians, African-Americans and Hispanics. These ethnically-differentiated versions were investigated in two studies, ${ }^{10,23}$ which found excellent levels of reliability ( 0.88 and 0.70 , respectively) for the different ethnicities studied.

Developmental factors (typical development vs. atypical development) and appropriateness to the age group of the child

The appropriateness of the pain scale with relation to developmental factors and age group of the child is an important aspect to be taken into account when considering these measurement instruments. Studies were carried out into the psychometric properties of scales used with children with special needs. ${ }^{16,19,24}$ It should be emphasized that studies of this nature are important, since they represent an advance in the development of instruments of pain assessment specific to populations of children with special needs. In studies of children with severe cognitive problems, the $\mathrm{PPP}^{16}$ and NCCPC- $\mathrm{R}^{19}$ were used in the context of assessment of painful situations and after surgical procedures, respectively. Only one study assessed the psychometric properties of the Pain Assessment Instrument Cerebral Palsy (PAICP), ${ }^{24}$ which is a pain assessment scale specifically for individuals with cerebral palsy.

Methodological precautions taken to ensure that specific scales used were appropriate to age group and developmental variations were an important criteria of some studies, specifically when the respondents were children themselves. The Oucher Faces Scale, 10,21,23 the Colored Analogue Scale $(\mathrm{CAS})^{11,21}$ and the FPS ${ }^{18}$ are examples of simple scales, which were the most used and which can be administered by children to assess their own pain from the age of 3 years. ${ }^{10,21,23}$

In order to attest to the compatibility between respondents' cognitive level and correct understanding of the scale, prior cognitive testing was an inclusion criterion of two studies in which children themselves were the respondents of pain self-assessment scales. ${ }^{19,24}$ In one study ${ }^{19}$ the Jean Piaget tests were administered in order to assess the cognitive level of children as an inclusion criterion, before the pain scales 
were used. The "seriation of blocks" test was also applied, in order to test the cognitive capacity of children to use the scales and to minimize possible errors in applying them. The Columbia Mental Maturity Scale (CMMS) was used with special populations of children with cerebral palsy, ${ }^{24}$ to test the mental level of children, as an inclusion criterion for study participants.

\section{Multiple informants/respondents}

Self-reporting consists of the ability of individuals to communicate their pain and, therefore, is the best indicator of the sensation of pain as a subjective experience. ${ }^{3}$ Indeed, it was found that the children themselves were the only respondents in five of the pain assessment studies. ${ }^{10-12,22,23}$ The five scales most used for self assessment of pain in children, in descending order, were as follows: the FPS, ${ }^{11,12,22}$ the Oucher Faces Scale, ${ }^{10,23}$ the CAS, ${ }^{11}$ the FAS ${ }^{11}$ and the Wong-Baker Faces Scale. ${ }^{23}$

The FPS was considered the most appropriate scale for self-assessment of pain intensity. ${ }^{11,12,22}$ The Wong-Baker Faces Scale ${ }^{23}$ should be used with caution, since the literature contains serious criticism of this scale because it includes a "smiley face" which is not necessarily related to a total absence of pain. ${ }^{3}$ Thus, an individual may not be in pain but may also not be happy, and therefore the "smiley face" would not correctly correspond to the "no pain" state.

In addition to self-reporting, when pain assessment is based on more than one informant it offers the advantage of promoting increased objectivity and minimizing the possible bias of a single assessment. Just one study combined children's self-reported assessments of pain with nurses' assessments. ${ }^{9}$ In another three studies, although there was no pain self-assessment by the children, pain assessments were carried out by two types of informant, parents and health professionals (physicians, nurses and physiotherapists). ${ }^{18,19,21}$

Due to the lack of verbal communication and/or difficulties in using self-reported pain assessments with special populations, pain assessment was carried out by other informants than the children themselves. In seven studies, pain assessment was carried out on the basis of a single informant: nurses, ${ }^{13-15,17}$ parents $^{20}$, trained observers ${ }^{8}$ or children's caregivers. ${ }^{19}$ Where pain assessments were carried out by more than one informant, these were carried out by parents and healthcare professionals ${ }^{16}$ and by nurses and independent observers. $^{7}$

\section{Measures of pain and stress}

Just one study used a combination of measures of self-reported pain, physiological measures, and measures of stress and discomfort in invasive procedures in situations of repeated blood collections. ${ }^{21}$ The Oucher Faces Scale and the
CAS were self- administered by children in pain and discomfort assessments; parents and nurses used the Visual Analogue Scale (VAS) to assess pain and fear and the degree of the children's cooperation during the invasive procedure. The BBDS was used by four independent observers who assessed the children's stress and discomfort during the procedure. Heart rate was used as a physiological measure of pain. The diversity and quantity of respondents and pain indicators assessed in this study assured greater objectivity of assessment, and also made it possible to compare the different assessment indicators. It was found that the BBDS, which combines indicators of stress and discomfort, exhibited excellent psychometric properties; however, as a multidimensional scale it provided a nonspecific pain assessment, since this was also combined with indicators of stress and discomfort.

\section{Studies of the application of pain measurement instruments}

\section{Considerations on the age group of the child}

Age proved to be an important variable to be considered in studies of pain assessment in children with typical development in different contexts. ${ }^{25-27}$ The FPS was used as a self-assessment pain scale with children from 3 to 7 years of age. ${ }^{25,26}$ It was found that the FPS was more accurate for pain self-assessment by children 6 and over than by children aged 3 to 5 years. ${ }^{25}$ Furthermore, it was found that children aged 5 to 7 years of age exhibited greater control over their verbal reactions to the pain they felt than children from 3 to 4 years of age, in a study of immunization (intramuscular injections). ${ }^{26}$ This is probably the result of the socialization process of children from 5 to 7 , who acquire greater self-control of their behavior and control of impulsivity, thereby allowing them to inhibit manifestations about pain felt in the presence of other people.

Perception of the pain of others by children was also investigated with relation to age differences and it was found that the older the child the more sensitive they were to the intensity of another's pain. ${ }^{27}$ These findings suggest there is a process of maturation of the ability to detect the pain of others, which, in turn, tends to vary according to age.

\section{Parental pain assessments of children in the postoperative context}

Parental assessment of children in the postoperative context and the administration/use of analgesia, using repeated measures, were the subject of three studies, ${ }^{28-30}$ two of which used the PPPM as their main instrument. ${ }^{28,30}$ This scale is a valid instrument for parental assessment of postoperative pain in children with typical development.

In general, studies that used the PPPM found that the greater the intensity of pain and the number of pain behaviors, the greater analgesic administration became. ${ }^{28,30}$ In 
addition to this, when assessments made by fathers were compared with assessments made by mothers, there were differences in relation to their perception of the child's analgesia; fathers considered that prescribing analgesics would have more prejudicial effects than mothers did.

Parental assessment proved to be sensitive in a study that compared pain assessments carried out by children's parents and by medical students in a surgical context. ${ }^{29}$ The study found a strong positive correlation between assessments by parents and students; furthermore, with relation to the parents' scores, these were sensitive to their children's pain symptoms since their scores were significantly higher before analgesia than afterwards.

\section{Relationship between pain and temperament}

One study investigated the relationship between temperament and pain, ${ }^{30}$ using the Behavioral Style Questionnaire (BSQ), filled out by parents, to evaluate the children's temperament; the PPPM was used to detect behavioral changes related to the experience of pain; the Wong-Baker Faces Scale was used as a self-assessment scale for the children. It was found that, with relation to the dimensions of temperament, children whose parents considered them to be more active and who had a more negative mood reported higher pain scores in hospital.

Pain assessment by parents and/or caregivers of children with special needs

\section{a) Facial expression as valid indicator of pain in special populations}

With relation to pain assessment in samples of children with cognitive problems, ${ }^{31}$ cerebral palsy ${ }^{32}$ and autism, ${ }^{33}$ the studies state that facial expression is a valid indicator which should be taken into consideration in pain assessment, not only for children with typical development, but also for children with special needs.

According to parents' perception of the pain behaviors of children with cerebral palsy, ${ }^{32}$ crying, as a facial expression, was considered to be the behavior most frequently exhibited by these children when expressing pain. Furthermore, children with autism exhibited similar facial activity to children without autism, in pain assessments during invasive procedures; nevertheless, the group of children with autism exhibited significantly more facial activity than the group without autism, but only during insertion of the needle. ${ }^{33}$

\section{b) Scales appropriate for special populations}

The use of specific instruments for pain assessment in special populations was an important methodological precaution observed in three studies. The Caregivers' Pain Survey (CPS), an instrument derived from the Non-Communicating Children's Pain Checklist (NCCPC) and the NCCPC itself were used to identify parents' perception is with relation to assessment of pain behaviors of children with cerebral palsy ${ }^{32}$ and in children with autism, ${ }^{33}$ respectively. The Pain Opinion Questionnaire ( $P O Q$ ), in turn, is an instrument that was specially developed to assess the pain of children with cognitive deficits. ${ }^{34}$

c) The importance of descriptive studies of pain using interviews

Given that it is not possible to use self-reporting with special populations, descriptive analysis studies are important, from a methodological point of view, since they constitute prerequisites for the identification of specific pain descriptors for pain assessment in these populations. Facial expression was found to be the most sensitive indicator out of 23 pain descriptors for post-surgical pain assessment in children with severe cognitive problems. ${ }^{31}$

One descriptive study was carried out using interviews with the parents of children with profound special needs, suffering from cerebral palsy and degenerative cerebral syndromes $^{35}$, the authors developed a thematic analysis of the verbal content of the interviews. This study identified the following principal themes related to assessment and management of pain in these children: Learning to live with the pain; Dealing with uncertainties and difficulties in identifying the child's pain; expression of pain, emphasizing facial expression; and Taking decisions; managing the child's pain and relationship with health professionals.

d) Pain assessment and stress in special populations

One important finding is related to pain assessment in children with autism during blood collection procedures, using specific Scales for the assessment of pain and stress administered to the parents. ${ }^{33}$ The CFCS and the Observational Scale of Behavioral Distress (OSBD) were used, respectively, to evaluate facial activity indicative of pain and stress behavior in children. It was found that the group of children with autism exhibited more responses to pain and stress during the post needle insertion and recovery phase when compared with the group without autism.

e) Underestimated assessments of the pain of children from special populations

Considering that pain self-assessment is not always possible in special populations, pain assessment of these populations carried out by caregivers may occur bias, since pain may be under or over estimated by examiners. The caregivers of children with severe cognitive deficits associate these children's sensation of pain with the severity of their cognitive deficits. They tend to underestimate the pain felt by children with mild or moderate cognitive deficits, since they believe that such children feel less pain than do children with typical development. ${ }^{34}$

In the study of children with autism, ${ }^{33}$ parents' assessments of the children's sensitivity and reactivity to pain did 
not correspond with pain-related facial activity and stress behaviors exhibited by the children with autism, despite the pain-related facial activity of the groups of children with and without autism having been similar. The parents tended to underestimate pain and did not take the children with autism facial expressions into account in their assessments. These findings show that children with autism are not indifferent or insensitive to pain, since they exhibited similar facial activities to the children without autism; nevertheless, the parents of the children with autism underestimated their pain assessments.

\section{Studies of evaluations of pain-relief intervention procedures}

Assessments of pain management interventions were the subject of four studies, ${ }^{36-39}$ which focused on acute pain. Two of these were studies of non-pharmacological interventions evaluating, respectively, minor painful invasive procedures i.e., blood collection ${ }^{36}$ and immunization ${ }^{37}$; while another two studies investigated pharmacological interventions, assessing postoperative pain. ${ }^{38,39}$

\section{Non-pharmacological interventions}

The non-pharmacological intervention studies investigated the effects of different strategies to cope with invasive procedures. Training intended to help children to deal with the pain of an immunization procedure proved ineffective without the help of trainers. ${ }^{37}$ This study, therefore, indicates the importance of parents and nurses being present as an important variable related to the efficacy of interventions for coping with painful procedures.

Using an experimental design comparing randomized groups, it was possible to test the efficacy of positioning and distraction strategies for dealing with pain, as assessed by the FPS; fear was assessed using the Glasses Fear Scale, and stress was assessed using the Procedure Behavioral Checklist (PBCL). ${ }^{36}$ During a blood collection procedure, children who were positioned by their parents close to the chest or sitting next to them and were distracted with children's storybooks had lower pain and fear assessment scores than a control group.

\section{Pharmacological intervention}

Although the pharmacological intervention studies 38,39 had different objectives, both were carried out with samples of children from birth until 3 years of age, stratified by age group. They used the following instruments to assess pain: the VAS and the COMFORT Scale Behavior, applied by the nursing team. Physiological indicators such as arterial blood pressure and heart rate were also assessed.

When investigating the association between physiological and behavioral measures of pain and identifying factors predictive of responses to pain, it was found that heart rate and arterial blood pressure correlated with the COMFORT scale scores. ${ }^{38}$ It should be pointed out that there was a large degree of individual variability in the babies' responses. The neonates exhibited the lowest VAS scores, when compared with the other three age groups ( 1 to 6,7 to 12 and 12 to 36 months). Furthermore, mean arterial blood pressure increased in line with the age of the children. On the other hand, the highest pain scores were observed among the youngest children. With relation to medication, the greater the pain the more morphine was given to the children, following a standard-protocol for dosages predefined for the study.

One study investigated the efficacy of two different standardized modes of administering morphine for postoperative analgesia (continuous vs. intermittent). ${ }^{39}$ No significant differences were found between the two forms of morphine administration for analgesia for postoperative pain in all sample children aged zero to 3 years after cases of abdominal or thoracic surgery. However, specifically in the 12 to 36 months age subgroup, continuous administration was more effective than intermittent.

\section{Pain assessment, fear and stress}

Another study evaluated pain, fear and stress associated with the invasive procedure of blood collection using specific and appropriate instruments to measure each construct separately. ${ }^{36}$ Pain was assessed using the FPS, fear using the Glasses Fear Scale and stress behaviors were assessed using the PBCL. A positive relationship was detected between the children's pain and fear assessments. Furthermore, the PBCL scores proved to be sensitive to the phases of pain assessment, such as: pre-procedure, during procedure and post-procedure.

\section{Discussion}

This review into assessment of the behavior of children in painful situations has shown that acute pain has been studied more than chronic pain, using measurement instruments the majority of which have ordinal scales. Acute pain has the marked characteristic of activating the organism, in order to bring it to a state of alert, which makes the expression of physiological and behavioral manifestations easier. Therefore it is more easily observed than chronic pain. In contrast, chronic pain tends to depress the organism's responses making observation of the manifestations of pain more difficult and making multiple resources necessary so that the progression of pain may be adequately assessed. ${ }^{1}$

The majority of the pain scales studied was of the one-dimensional type, and the dimension most often evaluated was the intensity of pain. This dimension specifically refers to the sensory aspect of pain, which, although very relevant to pain assessment, is not sufficient for complete understanding of the phenomenon of pain, which is multidimensional. The combined use of scales which assess at least two different dimension's should be adopted, considering the advantages for clinical assessment of children. One 
example is the advantage of being able to assess intensity and location of pain in the clinical-hospital context.

The psychometric parameter validity was more often assessed than the other parameters. Validity is the essential criterion for an instrument to be adopted for the measurement of pain and in order to identify its adequacy to the assessment situation for which a scale is to be adopted. If a scale has been validated for acute procedural pain or postoperative pain this should be taken into account when making a decision on the best scale to be adopted. Furthermore, if a scale has, in addition to validity, other proven psychometric parameters, the greater that instrument's power of measurement will be. In this review, the FLACC, TPPPS, CHEOPS, OPS and PPP scales stand out for their high quality psychometrics.

With relation to the use of self-assessment pain scales for children with typical development, the FPS is of note as the scale most used in a variety of contexts. The Oucher Faces Scale, which has also been used with this population, was identified by the review as the only scale offering specific versions for different ethnicities and, therefore, should be used in transcultural studies of pain assessment.

The FLACC scale was the most used for assessment of pain in children according to behavioral indicators observed by caregivers. The FLACC was developed to assess the acute pain of children with typical development during the postoperative period. ${ }^{6}$ However, in the studies reviewed here, use of the FLACC was not restricted to the postoperative phase. ${ }^{8}$ Furthermore, the FLACC was also used with preverbal children with typical development, ${ }^{15}$ and with children with atypical development. ${ }^{13,14}$

One review study of behavioral measures of pain assessment submitted certain scales to a rigorous review procedure and found their levels of clinical evidence. ${ }^{40}$ The FLACC was recommended for postoperative pain assessment, the CHEOPS for preoperative and postoperative pain assessment, and the PPPM for postoperative pain assessment on hospital discharge: three scales widely used for pain assessment, according to the levels of clinical evidence given. ${ }^{40}$ In contrast, the AHTPS and the TPPPS were scales whose levels of evidence compromise their clinical use. One should therefore be cautious with relation to their clinical applicability.

Advances were observed with relation to pain assessment in children with atypical development, since self reporting of pain is difficult with such populations. This review found scales developed specially for pain assessment in special populations. The NCCPC-R, for example, assesses physiological and behavioral indicators in children with severe cognitive deficits; the PPP assesses pain in these same populations, although only using behavioral indicators; and the PAICP was specifically developed for pain assessment of children with cerebral palsy.
These studies show that, although these populations have similar facial activity to children with typical development, they are assessed by parents and caregivers with low sensitivity and reactivity to pain. One possible explanation is that parents and/or caregivers believe that children with special needs are less sensitive or feel less pain than children with typical development and, because of this, tends to underestimate the pain of children with special needs in their evaluations.

Facial expression was an important indicator, both in self-assessment pain scales in populations with typical or atypical development and in evaluations carried out by parents, caregivers or health professionals. Other studies have also indicated that facial expressions have an important function in pain assessment since they are one of the most reliable behavioral indicators of pain. ${ }^{3,40}$

Future studies of pain assessment during painful procedures for immunization and blood collection should consider that the use of certain strategies, which have been indicated in this review, may contribute to reducing pain. The presence of adults, whether the children's own parents or professionals from the health team, positioning of the child close to their parents' chest or seated at their side and children's storybooks used as a distraction strategy were significant variables for reducing pain and should be considered in studies of non-pharmacological interventions.

One limitation of the present study is related to identification of the psychometric properties of instruments. In this review it was possible to identify some of the psychometric parameters which were found by assessment of the properties of the target-scales of the studies. Other parameters relating to these target-scales, however, may appear in studies prior to the period of 2001 to 2006 . The FPS, for example, is a scale that is often used for pain assessment in the literature. In this review it was found that this scale meets the validity parameter, but this does not mean that, in prior studies, the scale has not met other psychometric parameters, such as reliability, sensitivity, specificity or applicability. This study has been used in studies of concurrent validity of new scales, which definitely suggests it has good psychometric qualities.

Considering the applicability of these scales in Brazil, it is opportune to emphasize the need for studies into the translation and adaptation of pain measurement instruments. As far as is known, few scales have been translated into Portuguese (Brazil), as have, for example, the FPS ${ }^{41}$ and the Wong-Baker Faces Scale. ${ }^{42}$ It is, therefore, recommended that future studies deal both with the issue of translation into Portuguese and adaptation and with analysis of the clinical applicability of pain assessment instruments in clinical-hospital contexts.

\section{References}

1. Merskey $\mathrm{H}$, Albe-Fessard DG, Bonic J]. Pain terms: a list with definitions and notes on usage: recommended by the International Association for Study of Pain (IASP) Subcommitee on Taxonomy. Pain. 1979;6:249- 52 
2. Puccini RF, Bresolin AMB. Dores recorrentes na infância e na adolescência. J Pediatr (Rio J). 2003;79 Supl. 1:S65-S76.

3. Chambers CT, McGrath PJ. Pain measurement in children. In: Ashburn MA, Rice LJ, editores. Management pain. New York: Churchill Livingston; 1998. p. 625-34.

4. Walco GA, Conte PM, Labay LE, Engel R, Zeltzer LK. Procedural distress in children with cancer: self-report, behavioral observations, and physiological parameters. Clin J Pain. 2005; 21:484-90.

5. Fitzgerald M. Developmental biology of inflammatory pain. Br J Anaesth. 1995;75:177-85.

6. Silva JA, Ribeiro-Filho MP. Avaliação e mensuração de dor-pesquisa, teoria e prática. Ribeirão Preto: Furple; 2006.

7. Suominen P, Caffin C, Linton S, McKinley D, Ragg P, Davie G, et al. The cardiac analgesic assessment scale (CAAS): a pain assessment tool for intubated and ventilated children after cardiac surgery. Paediatr Anaesth. 2004;14:336-43.

8. Suraseranivongse S, Santawat U, Kraiprasit K, Petcharatana S, Prakkamodom S, Muntraporn N. Cross-validation of a composite pain scale for preschool children within 24 hours of surgery. Br J Anaesth. 2001;87:400-5.

9. Hartrick CT, Kovan JP. Pain assessment following general anesthesia using the Toddler Preschooler Postoperative Pain Scale: a comparative study. J Clin Anesth. 2002;14:411-5.

10. Beyer JE, Turner SB, Jones L, Young L, Onikul R, Bohaty B. The alternate forms reliability of the Oucher pain scale. Pain Manag Nurs. 2005;6:10-7.

11. Perrott DA, Goodenough B, Champion GD. Children's ratings of the intensity and unpleasantness of post-operative pain using facial expression scales. Eur ] Pain. 2004;8:119-27.

12. Bosenberg A, Thomas J, Lopez T, Kokinsky E, Larsson LE. Validation of a six-graded faces scale for evaluation of postoperative pain in children. Paediatr Anaesth. 2003;13: 708-13.

13. Voepel-Lewis T, Merkel S, Tait AR, Trzcinka A, Malviya S. The reliability and validity of the face, legs, activity, cry, consolability observational tool as a measure of pain in children with cognitive impairment. Anesth Analg. 2002;95:1224-9.

14. Voepel-Lewis T, Malviya S, Tait AR. Validity of parent ratings as proxy measures of pain in children with cognitive impairment. Pain Manag Nurs. 2005;6:168-74.

15. Manworren RC, Hynan LS. Clinical validation of FLACC: preverbal patient pain scale. Pediatr Nurs. 2003;29:140-6.

16. Hunt A, Goldman A, Seers K, Crichton N, Mastroyannopoulou K, Moffat $\mathrm{V}$, et al. Clinical validation of the paediatric pain profile. Dev Med Child Neurol. 2004;46:9-18.

17. Stewart B, Lancaster G, Lawson J, Williams K, Daly J. Validation of the Alder Hey Triage Pain Score. Arch Dis Child. 2004;89: 625-30.

18. Breau LM, McGrath PJ, Craig KD, Santor D, Cassidy KL, Reid GJ. Facial expression of children receiving immunizations: a principal components analysis of the child facial coding system. Clin J Pain. $2001 ; 17: 178-86$.

19. Breau LM, McGrath PJ, Camfield CS, Finley GA. Psychometric properties of the non-communicating children's pain checklist-revised. Pain. 2002;99:349-57.

20. Kokki A, Kankkunen P, Pietila AM, Vehvilainen-Julkunen K. Validation of the Parents' Postoperative Pain Measure in Finnish children aged 1-6 years. Scand J Caring Sci. 2003;17:12-8.
21. Tucker CL, Slifer KJ, Dahlquist LM. Reliability and validity of the brief behavioral distress scale: a measure of children's distress during invasive medical procedures. J Pediatr Psychol. 2001;26: 513-23.

22. Hicks CL, von Baeyer CL, Spafford PA, van Korlaar I, Goodenough B. The Faces Pain Scale-Revised: toward a common metric in pediatric pain measurement. Pain. 2001;93:173-83.

23. Luffy R, Grove SK. Examining the validity, reliability, and preference of three pediatric pain measurement tools in African-American children. Pediatr Nurs. 2003;29:54-9.

24. Boldingh EJ, Jacobs-van der Bruggen MA, Lankhorst GJ, Bouter LM. Assessing pain in patients with severe cerebral palsy: development, reliability, and validity of a pain assessment instrument for cerebral palsy. Arch Phys Med Rehabil. 2004;85: 758-66.

25. Stanford EA, Chambers CT, Craig KD. The role of developmental factors in predicting young children's use of a self-report scale for pain. Pain. 2006;120:16-23. Epub 2005 Dec 13.

26. Stanford EA, Chambers CT, Craig KD, McGrath PJ, Cassidy KL. "Ow!": spontaneous verbal pain expression among young children during immunization. Clin J Pain. 2005;21:499-502.

27. Deyo KS, Prkachin KM, Mercer SR. Development of sensitivity to facial expression of pain. Pain. 2004;107:16-21.

28. Kankkunen $P$, Vehvilainen-Julkunen $K$, Pietila AM, Kokki H, Halonen P. Parents' perceptions and use of analgesics at home after children's day surgery. Paediatr Anaesth. 2003;13:132-40.

29. Morgan J, Peden V, Bhaskar K, Vater M, Choonara I. Assessment of pain by parents in young children following surgery. Paediatr Anaesth. 2001;11:449-52.

30. Helgadóttir HL, Wilson ME. Temperament and pain in 3 to 7-year-old children undergoing tonsillectomy. J Pediatr Nurs. 2004;19:204-13.

31. Terstegen C, Koot HM, de Boer JB, Tibboel D. Measuring pain in children with cognitive impairment: pain response to surgical procedures. Pain. 2003;103:187-98.

32. Hadden $\mathrm{KL}$, von Baeyer, CL. Pain in children with cerebral palsy: common triggers and expressive behaviors. Pain. 2002;99: 281-8.

33. Nader R, Oberlander TF, Chambers CT, Craig KD. Expression of pain in children with autism. Clin J Pain. 2004;20:88-97.

34. Breau LM, MacLaren J, McGrath PJ, Camfield CS, Finley G.A. Caregivers' beliefs regarding pain in children with cognitive impairment: relation between pain sensation and reaction increases with severity of impairment. Clin J Pain. 2003;19: 335-44.

35. Carter B, McArthur E, Cunliffe M. Dealing with uncertainty: parental assessment of pain in their children with profound special needs. J Adv Nurs. 2002;38:449-57.

36. Cavender K, Goff MD, Hollon EC, Guzzetta CE. Parents' positioning and distracting children during venipuncture. Effects on children's pain, fear, and distress. J Holist Nurs. 2004;22: 32-56.

37. Cohen LL, Bernard RS, Greco LA, McClellan CB. A child-focused intervention for coping with procedural pain: are parent and nurse coaches necessary? J Pediatr Psychol. 2002;27:749-57.

38. van Dijk M, de Boer JB, Koot HM, Duivenvoorden HJ, Passchier J, Bouwmeester $\mathrm{N}$, et al. The association between physiological and behavioral pain measures in 0- to 3-year-old infants after major surgery. J Pain Symptom Manage. 2001;22:600-9. 
39. van Dijk M, Bouwmeester NJ, Duivenvoorden HJ, Koot HM, Tibboel D, Passchier J, et al. Efficacy of continuous versus intermittent morphine administration after major surgery in 0-3-year-old infants; a double-blind randomized controlled trial. Pain. 2002;98:305-13.

40. von Baeyer CL, Spagrud LJ. Systematic review of observational (behavioral) measures of pain for children and adolescents aged 3 to 18 years. Pain. 2007;127:140-50.

41. Bieri D, Reeve R, Champion GD, Addicoat L, Ziegler J. The Faces Pain Scale for the self-assessment of the severity of pain experienced by children: Development, initial validation and preliminary investigation for ratio scale properties. Pain. 1990; 41;139-50.
42. Elsevier. Wong on web. [Internet] Translations of Wong-Baker faces pain rating scale. http://www.mosbysdrugconsult.com/ WOW/facesTranslations.html. Access: 22/06/2008.

Correspondence:

Maria Beatriz Martins Linhares

Faculdade de Medicina de Ribeirão Preto - USP

Campus Universitário Monte Alegre

Prédio da Saúde Mental

Av. Tenente Catão Roxo, 2650/52

CEP 14048-900 - Ribeirão Preto, SP - Brazil

Tel. : +55 (16) 3602.4610

E-mail: luleonetti@hotmail.com, linhares@fmrp.usp.br 